مهارة الكلام في درس اللغة الألمانية

مساهمة في دعم مهارة الكلام في درس اللغة الألمانية كلغة اجنبية

\author{
م. د. قاسم جبر عبرة \\ قسم الترجمة / كلية الاداب جامعة واسط عبرن \\ qebrah@uowasit.edu.iq
}

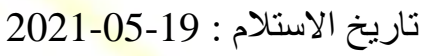

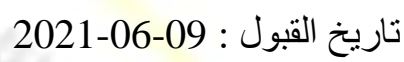

الخلاصة

الهذف من هذه الورقة البحثية هو الثروع في بحث مهارة الكلام عند طلاب اللغة الألمانية كلغة أجنبية في كلية اللغات و اقتر اح تدابير علاجية في شكل بر امج تعليمية. بعد أن حددت النقص ومشاكل مهارة الكلام لدى لهى طلاب قسم اللغة الألمانية بكلية اللغات من خلال اجراء استبيان، قررت في هذه الورقة البحثية تعويض ومعالجة هذا النقص، وذللك من خلال تقديم مقترحات حول استخدام برمجيات التواصل التربوية. من أجل إضفاء الثرعية على هذا العمل، قمت بإجر اء استبيان لـ 54 من طلاب المرحلة الرابعة والثالثة في قسم اللغة الألمانية في كلية اللغات في بغداد لمعرفة مستوى مهارة الحديث لديهم وللحصول على إجابة لسؤ الي عما إذا كان المستوى اللغوي في مهارة الكلام لاربع سنوات منو افقًا مع مهارتهم في الحديث. اذ تبين من خلال إجاباتهم على الأسئلة الموجهة إليهم عبر منصة جوجل كلاس روم، أن لايهم بالفعل مشكلة في القدرة على التحدث و الفهم. وبعد تحليل المتطلبات والتدابير التعليمية لتحسين مهار ات التحدث لدى الطلاب، قمت بتقديم مقترحات حول استخدام برامج تو اصلية لتحسين أوجه القصور في مهارة الكلام وتبرير قيمتها التعليمية.

كلمات مفتاحية: مهارة الكلام، بر امج تعليمية، القيمة التعليمية 


\title{
Die Sprechfertigkeit im DaF-Unterricht
}

Sprachlicher Beitrag zur Förderung der Sprechfertigkeit im DaF-Unterricht

\author{
Dr. Lec.Qasim J. Abrah \\ Translation Department /College of Arts \\ Wasit University
}

Receipt date: 2021-05-19

Date of acceptance: 2021-06-09

Abstract

Das Ziel dieses Beitrags ist es, die mündliche Kompetenz bei Germanisten der Sprachenfakultät in Bagdad zu untersuchen und didaktische Maßnahmen in Form von Lernsoftware für die Komoetenzverbesserung zu entwickeln. Nachdem ich Defizite bei den Studierenden in der Sprechfertigkeit über eine Befragung festgestellt hatte, entschied ich mich im Folgenden, diese Defizite durch den Einsatz von kommunikationspädagogischer Software zu kompensieren.

Um diese Arbeit zu legitimieren, führte ich eine Befragung von 54 Studierenden im dritten und vierten Studienjahr durch, um ihr Sprachniveau in der Sprechfertigkeit herauszufinden und eine Antwort auf meine Frage zu erhalten, ob die für vier Studienjahre angestrebte mündliche Sprachfähigkeit sich mit ihrer tatsächlichen Sprechfähigkeit deckt. Aus ihren Antworten auf die Fragen, die über Google Classroom an sie gerichtet wurden, ging hervor, dass sie tatsächlich Probleme im mündlichen Verstehen und Sprechen haben. Nach einer Analyse der didaktischen Anforderungen und Maßnahmen zur Verbesserung der Sprechfertigkeiten der Studierenden habe ich Maßnahmen zur Verbesserung dieser Fähigkeiten entworfen, beschrieben und deren didaktischen Wert begründet.

Schlüsselwörter: Sprechfertigkeit, Lernsoftware, didaktischer Wert 


\section{Terminologie}

Sprechen ist neben Hören, Lesen und Schreiben eine der klassischen ,vier Fertigkeiten“, die im Sprachunterricht entwickelt werden sollen (vgl. Krumm et al. 2010: 983). .Der Begriff Sprechfertigkeit fasst einzelne Teilkompetenzen zusammen, die die Lernenden erreichen können. Er muss in der Lage sein, Gesprächskontakte herzustellen und zu halten. Er muss wissen, wie er ein Gespräch über ein beliebiges Thema beginnt, wie er es entsprechend fortsetzt und letztendlich korrekt beendet.

Darüber hinaus muss ein Lernender in der Lage sein, seine Sprechintentionen mit Hilfe eines kohärenten Sprachgebrauchs zu realisieren. Denn er muss z. B. bei einem Gespräch mit einer anderen Person in der Lage sein, sprachliche Mittel zur Gesprächsführung in seinem Gedächtnis abzurufen und angemessen einzusetzen.

\section{Durchführung der Erhebung}

\section{Forschungsstand}

Man betrachtet die Sprechfertigkeit als das größte Problem beim Erlernen von Fremdsprachen und so auch von der deutschen Sprache. Man stellt fest, dass ein Lernender fortgeschrittene Sprachkenntnisse hat und einen großen Bestand an Wörtern und Vokabeln besitzt. Wenn er jedoch spricht, bekommt man einen Eindruck, als wäre er ein Anfänger, und wenn er Sätze bildet, kann er sich die Wörter dafür nicht ins Gedächtnis zurückrufen. Auch wenn sein Gedächtnis ihm entsprechende Wörter liefert, stottert er und verzögert in der Aussprache. Was ist der Grund dafür? Wir werden uns nicht mit der theoretischen Diskussion zu diesem Thema befassen, sondern wir wollen praktische Schritte diskutieren. Kurz gesagt, der Hauptgrund für die Verzögerung der Sprechfertigkeit ist, dass die Sprache immer noch im Bewusstsein ist, während sie in das automatische Unterbewusstsein übertragen werden muss, um fließend zu werden und ohne daran eine Weile denken zu brauchen. So werden bereits gefertigte und konservierte Sätze verlangt, anstatt sie im Kopf gebildet werden zu müssen. Dies erfordert ein kontinuierliches Üben des Zuhörens und Sprechens.

„Die Sprache gehört zu den bedeutendsten geistigen Leistungen des Menschen. Die Verwendung von Sprachen vollzieht sich beim gesunden Menschen hoch automatisiert, so dass in den meisten alltäglichen Kommunikations und Sprachverstehensprozesse ohne Schwierigkeiten ablaufen. Erst wenn es in bestimmten Situationen zu besonderen Belastungen kommt, geraten z.B. akustische oder sprechmotorische Problerme ins Bewusstsein“" (Grohnfeldt 2007: 332).

Das Zuhören ist einfach und leicht zu üben. Es gibt so viele Quellen, darunter auch digitale wie Websiten und Apps, die einem Lernenden Tausende von Audiostunden anbieten, womit man die deutsche Sprache authentisch hören kann. Das Problem liegt aber nicht daran, sondern am Sprechen, insbesondere, wenn man nicht in einem Zielsprachenland lebt. So stellt sich die Frage: Wie üben wir, Deutsch zu sprechen? 


\section{Sprechfertigkeit als Lermziel im Fremdsprachenunterricht}

Bei der Grammatik-Übersetzungsmethode ist das Sprechen nicht weiter der Rede wert. Das Gespräch konzentrierte sich schon früh auf praktische Ansätze, die auf den Sprachgebrauch abzielten. Diese Ansätze werden heute wieder nutzbar gemacht. Die neolinguistische Reformbewegung konzentrierte sich hauptsächlich auf die Aussprache. Die Ergebnisse der neu etablierten wissenschaftlichen Phonetik wurden aufgegriffen. Die technische Hilfe war das Sprachlabor. Das Sequenzhören wurde als Muster-Übung im Dienst der Automatisierung grammatikalischer Strukturen verwendet. Im kommunikativen Sprachunterricht scheint die Weiterentwicklung des Sprechens eine selbstverständliche Angelegenheit zu sein und bedarf keiner Rechtfertigung. In anderen Bereichen des globalen Lernens wird jedoch ein anderer Schwerpunkt auf das Sprechen gelegt.

\section{Forschungsproblem}

Entspricht die während der Lern- und Studierenzeit erworbene Sprechfertigkeit dem sprachlichen erreichten Niveau der Studierenden vom vierten Studienjahr im Fachbereich Deutsch als Fremdsprache? Als Testpersonen wurden die Studierenden des vierten Studienjahres, d.h. des letzten Studienjahrs, in Betracht gezogen, weil sie sich in der Endstufe befinden und fast das gesamte Programm von vier Studienjahren vollzogen und das hierfür festgelegte Niveau B1. erreicht haben sollten. Für diese Studie wurden 54 Studierende aus der Deutschabteilung an der Sprachenfakultät Universität Bagdad befragt.

Dieser Beitrag befasste sich mit dem allgemeinen Problem der unzureichende Entwicklung der Sprechfähigkeiten im DaF-Unterricht, darüber hinaus mit der Verwendung einer Lernsoftware zur Förderung der Sprechfähigkeit bei Studierenden an der Deutschabteilung an der Sprachenfakultät im Irak. Die Hauptfragen sind, wie man dieses Defizit an Sprechübungen im Deutschunterricht verringern und mehr Lernmöglichkeiten für Studierende auf stressfreie und motivierte Methode anbieten kann. Ausgangspunkt dieser Forschung sind die Hypothesen, wonach die Verwendung einer Kommunikationslernsoftware eine Kompensationsmöglichkeit sein könnte. Der Einsatz neuer Medien würde dazu beitragen, die Motivation der Sudierenden zu steigern.

\section{Fragebögen}

Die Fragebögen beeinhalten gegliederte Fragen mit unterschiedlichen Erkenntniszielen. Gemäß der Fragebögen sollen die Studierenden ihr Nievau in der Sprechfähigkeiten einschätzen und wie sie an Sprechaktivitäten innerhalb und außerhalb dem Unterricht teilnehmen.

\section{Fragebogenauswertung}

Die 1. Frage: Ich kann Personen und Orte beschreiben und kann mich verständigen, wenn eine andere Person langsam spricht. (Ich kann alltägliche Ausdrücke und ganz einfache Sätze verstehen)

Diagramm 1 


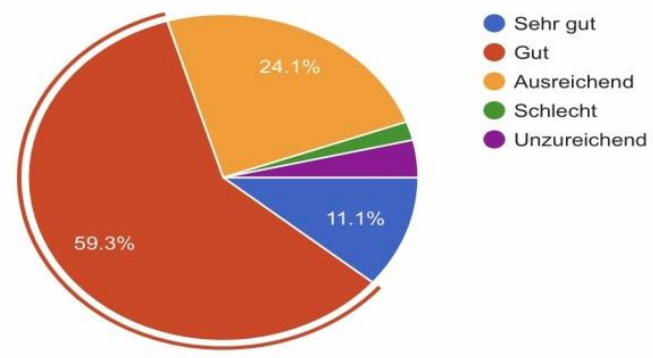

Diagramm 1 verdeutlicht die Anzahl der Personen, denen die Fragen des Fragebogens zur Selbsteinschätzung ihres Sprachniveaus gestellt wurden. Ihre Antworten variieren zwischen sehr gut und unzureichend. Es gibt jenige, die mit ihrem Sprachniveau zufrieden sind, und andere, die nicht zufrieden sind.

Die 2. Frage: Ich kann ein sehr kurzes persönliches Gespräch beginnen, aber ich kann es nicht weiterführen und beenden, weil ich nicht genug verstehe.

Diagramm 2

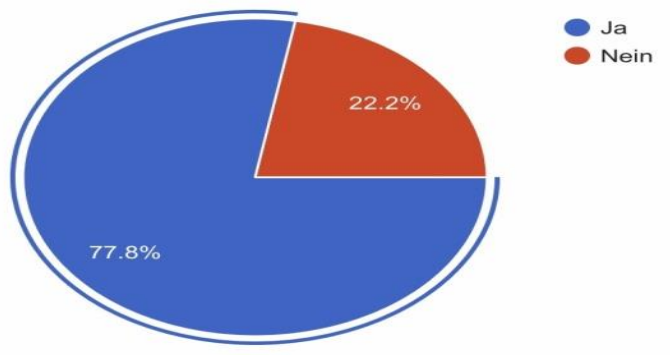

Diagramm 2 zeigt, dass 77.8\% Prozent der Studierenden nicht in der Lage sind, ein weiteres Gespräch weiterzuführen und zu beenden.

Die 3. Frage: Ich kann Träume, Hoffnungen und Ziele beschreiben, eine Geschichte erzählen und mich ohne Vorbereitung an Gesprächen über vertraute und interessante Themen beteiligen.

Diagramm 3 


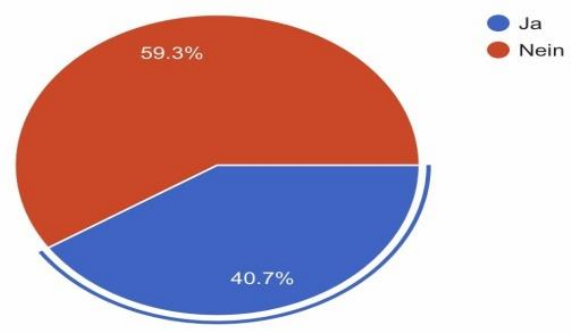

Diagramm 3 verdeutlicht, dass 59.3\% Prozent der Studierenden unfähig sind, ihre Träume, Hoffnungen und Ziele erfüllen.

Die 4. Frage: Ich kann mich aktiv an längeren Gesprächen über die meisten Themen beteiligen und dabei einige komplexe Satzstrukturen verwenden, ohne blitzschnell nach Worten zu suchen.

Diagramm 4

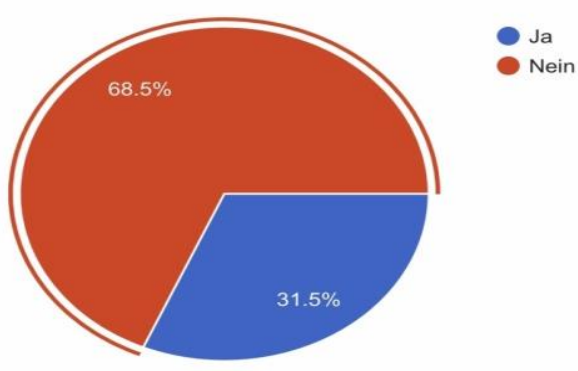

Diagramm 4 erklärt, dass $68.5 \%$ Prozent der Studierenden unfähig sind, sich an längeren Gesprächen über die meisten Themen zu beteiligen und dabei einige komplexe Satzstrukturen zu verwenden, ohne ständig wieder nach Worten zu suchen.

Die 5. Frage: Haben Sie schwierigkeiten beim Umgang mit sozialen Medien? 


\section{Diagramm 5}

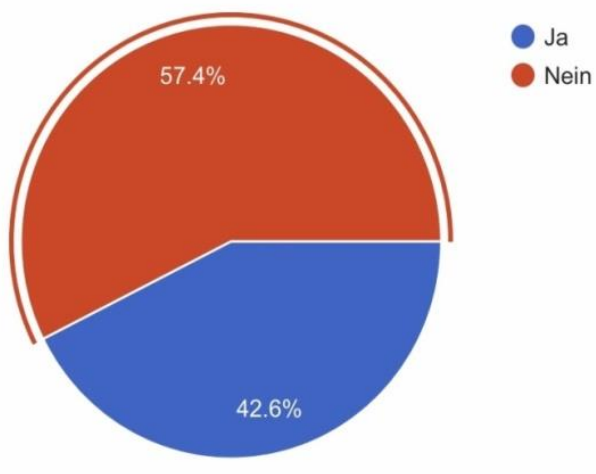

Diagramm 5 verdeutlicht, dass 57.4\% Prozent der Studierenden keinen Umgang mit sozialen Medien haben.

Die 6. Frage: Hören Sie Deutschlandfunk Nachrichten ?

Diagramm 6

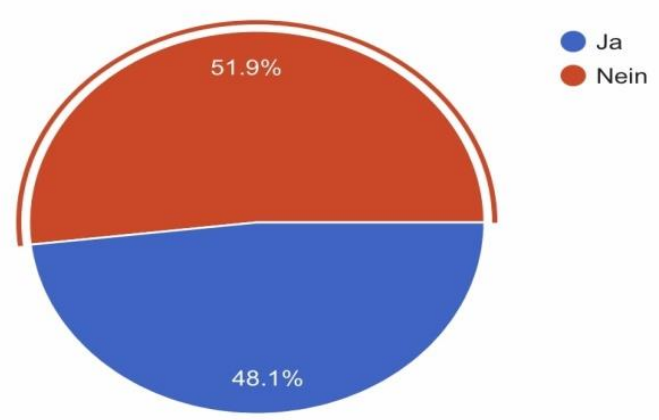

Diagramm 6 erklärt, dass 51.9\% Prozent der Studierenden Deutschlandfunk Nachrichten nicht hören.

Die 7. Frage: Wie oft folgen Sie Tagesnachrichten und Hintergrundberichten auf Deutsch?

Diagramm 7 


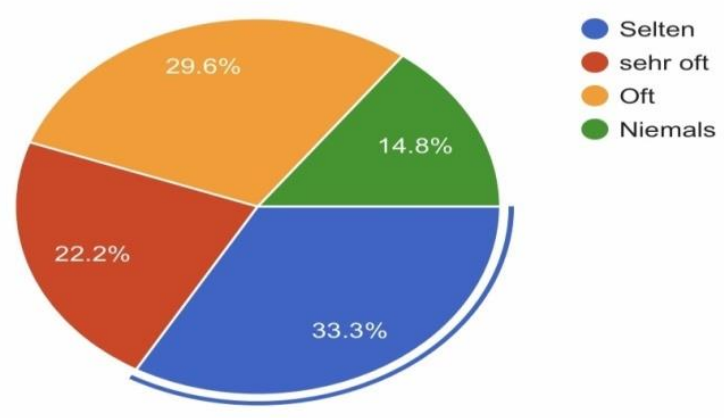

Diagramm 7 verdeutlicht, dass ein Großteil der Studierenden selten bis niemals Tagesnachrichten und Hintergrundberichten auf verfolgt. Es gibt diejenige, die selten, sehr oft, oft und niemals Tagesnachrichten und Hintergrundberichten auf Deutsch folgen. Der größte Teil der Studierenden folgen so selten Tagesnachrichten und Hintergrundberichten auf Deutsch.

Die 8. Frage: Unterhalten Sie mit deutschsprachigen Personen im In- und Ausland?

Diagramm 8

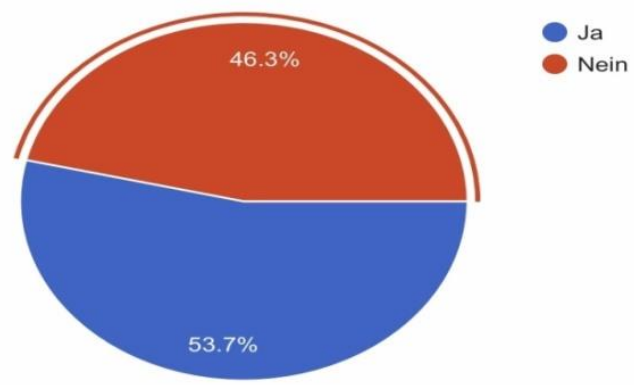

Diagramm 8 beschreibt, dass sich 46 Prozent der Studierenden nicht mit deutschsprachigen Personen im In-und Ausland unterhalten.

Die 9. Frage: Unterhalten Sie mit Ihrem Partner/Ihrer Partnerin im Klassenraum, in der Pause oder außerhalb der Universität auf Deutsch?

Diagramm 9 


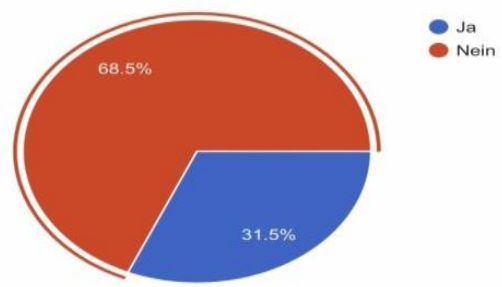

Diagramm 9 erklärt, dass sich 68 Prozent der Studierenden mit ihrem Partner/ihrer Partnerin im Klassenraum, in der Pause oder außerhalb der Universität auf Deutsch unterhalten.

\section{Diskussion der Ergebnisse}

Die Ergebnisse der durchgeführten Analyse werden im Folgenden zusammenfassend diskutiert.

Die Auswertungen der Fragebögen zeichnen folgendes elementare Bild. Die Ergebnisse der Fragebögen, die den Studierenden vorgelegt wurden, zeigten, dass die meisten Studierenden unter einem echten Problem in der Sprechfähigkeit leiden. Der Fragebögen zeigten auch, dass die meisten Studierenden keine Kommunikationsprogramme verwenden, um ihre Sprachkenntnisse, insbesondere das Sprechen, zu entwickeln.

\section{Einsatzmöglichkeiten bzw. Unterrichtsformen mit neuen Medien}

\section{Wie üben wir das Sprechen auf Deutsch?}

Dieser Beitrag bietet ein paar Tipps, beste Ratschläge und praktische Methoden, die für das Üben des Sprechens nützlich sind. Dies ist ein Ergebnis langjähriger Erfahrung auf diesem Gebiet.

\section{Das intensive Zuhören}

Man geht davon aus, dass Sprechen in hohem Maß vom Hören abhängt. Jeder hat schon einmal erlebt, wie Kinder im frühen Kleinkindalter sehr viel Zeit beim Zuhören, Beobachten verbringen und dann beginnen sie zu sprechen. Dieses intinsive Zuhören führt dazu, dass Sätze und Ausdrücke einem Empfänger in Kopf vertraut sind. Wenn der Verstand einen Satz immer wieder und auch wieder in verschiedenen Kontexten hört und sobald man sich in einer ähnlichen Situation befindet, wird man diesen Satz aussprechen, ohne über dessen Wörter und Syntax nachzudenken

Es ist unbestritten, dass es ohne Hören kein sinnvolles Sprechen im mündlichen Miteinander gibt und dass das verstehende Hören auch ,als wesentliche Voraussetzung für das richtige Lesen und Schreiben" gilt (Alhachami 2016:61). 
Man sollte so viel wie möglich zuhören, auch wenn man nichts davon versteht. Das klingt bestimmt ziemlich langweilig. Doch sollte man nicht leichtfertig aufgeben. Um Spaß beim Hören $\mathrm{zu}$ haben und die Langweile $\mathrm{zu}$ vermeiden, sollte man versuchen, Quellen und Dateien auszuwählen, die seinem Niveau entsprechen sollen, bis man etwas davon versteht. Gleichzeitig muss man weiter lernen und jeden Tag neue Wörter im Gedächtnis behalten, da das alleinige Zuhören ohne Auswendiglernen neuer Wörter nicht von großer Bedeutung ist, denn das Zuhören dient dazu, diese Wörter im Gedächtnis auf praktische Weise und in verschiedenen Sätzen und Kontexten zu verwenden.

Natürlich gibt es so viele Quellen und Audiodateien. Wir werden diese Audioquellen hier nicht auflisten, da dies den Rahmen sprengen würde. Wir werden später jedoch darauf zurückkommen, wie man entsprechenden Audiodateien für alle Sprachebenen erhalten kann, aber hier möchte ich zwei Quellen vorstellen, die die besten kostenlosen Quellen im Internet und Mobiltelefone sind:

1. Nicos Weg ist eine Serie, die aus 76 Folgen besteht, wobei man fast die vier Fertigkeiten (Sprechen, Hören, Lesen und Schreiben) fördern kann. „Nico ist Spanier und kommt nach Deutschland. Er kann aber kaum Deutsch sprechen“. Jede Folge besteht aus einem kurzen Dialog zwischen ein paar Personen über verschiedene Themen (sich und die anderen vorstellen). Diese Serie ist im Internet kostenlos verfügbar. Man kann die Folgen über eine Website oder über Android und iOS -App verfolgen.

\section{Die andere kostenlose Quelle ist eine Reihe von 10 Netflixserien: Dark}

Türkisch für Anfänger, Bad Banks, Babylon Berlin, Charité, Dogs of Berlin, Berlin Station, Stromberg, Weissensee und Parfum. Diese Serien umfassen verschiedene Themen, die interessante und aktuelle Sachen behandeln. Sie eignen sich eher für diejenigen die in der Zwischenphase oder fortgeschrittenen Kenntnissen der Sprache sind. Sie sind erreichbar über Webseiten im Netz oder über die inoffizielle Anwendung auf Android und iOS.

\section{Laut vorlesen}

Meistens lernen wir die deutsche Sprache aus verschiedenen Quellen, sei es über das Internet, Bücher oder andere Mittel, die lesbares Material bereitstellen. Es ist dabei sehr wichtig, dass man einen deutschen Text laut liest. Man sollte sich vorstellen, dass man auf einer Plattform stünde und diesen Artikel einer großen Menge von Publikum vorläse, als ob man das Mikrofon hälte, das in einem Saal voller Besucher widerhallt. An diesem Punkt muss man mit klarer und verständlicher Stimme sprechen. Man sollte sich daran gewöhnen, in einem hörbaren und klaren Ton zu lesen. Diese Angewohnheit dient dazu, die Sprechfertigkeit zu entwickeln und zu verbessern, insbesondere Wörter richtig auszusprechen.

„Die Lernenden sollen von Anfang an die Worte richtig aussprechen. Entweder während des Vokabelpaukens oder wärend des Unterrichts (die Zielsprache vom ersten Augenblick an sprechen). Diese Methode wird zwar als besonders „modern“ angesehen, ist aber nicht gehirngerecht. Beachten Sie, dass Babys der Sprache monatelang zuhören, bevor sie versuchen, die Klänge nachzuahmen, die sie so und so oft gehört haben, während wir von Sprachenlernen 
erwarten, dass sie sofort Klänge nachahmen, mit denen sie noch gar nicht vertraut sind“" (Birkenbihl 2013:195).

\section{Das Medium Film im Deutschunterricht}

Deutschlernen durch deutsche Filme ist eine der effektivsten Methoden des Lernens. Das Filmanschauen bietet eine Möglichkeit, Sätze und Ausdrücke, die in einem Film vorkommen, zu wiederholen. Man gebraucht eine Indoktrination-Methode, wenn Schüler/innen in den ersten Klassen unterrichtet werden. Diese Methode könnte ein erfolgreichster Ansatz sein, um die richtige Aussprache zu lernen.

„Der Umgang mit Film und Fernsehen kann zur Förderung und zum Erwerb zahlreicher Kompetenzen beitragen, die in den Bildungsstandards für das Fach Deutsch festgelegt sind und muss sich nicht zum Nachteil anderer Aufgaben des Deutschunterrichts auswirken“ (Staiger 2014: 248).

Dieser Lernstil besteht darin, dass man nach jedem Satz stoppt, den ein Schauspieler in einem Film sagt, und dieser Satz übersetzt oder gut verstanden wird. Dann spricht ein Lernende mehrmals diesen Satz nach und versucht, jedes Wort und die Schauspielerstimme nachzuahmen. Dieser Vorgang wird mehrmals wiederholt. So könnte diese Methode deswegen effektiv sein, weil man einen authentischen Sprechtext nachspricht und die Wörter einer Zielsprache richtig ausgesprochen werden, so könnte man Wörter und Phrasen richtig aussprechen lernen. (So könnte ein Lernender gerade auch Animationsfilme anschauen, da die Sprache dabei klar und gehoben ist).

\section{Finden Sie einen Begleiter, mit dem Sie sprechen können}

Diese Art vom Lernen gilt gilt als wichtigster und effektivster Weg, um das Sprechen zu auszuüben. Diese Methode bezieht sich darauf, dass man jemanden in seiner Nähe findet, der in einem ähnlichen Kurs ist oder besseres Niveau hat.

Sprechfertigkeit in einer Fremdsprache ist für den, der sie besitzt, praktisch verwendbar und verwertbar nur dann, wenn er mit Angehörigen der betreffenden in praktischen Verkehr tritt. Nur in diesem Falle wird die Sprechfertigkeit ein lebendiges Gut, sonst ist sie ein totes Kapital.

Man sollte unbedingt mit jemandem unter allen Umständen nur auf Deutsch ansprechen.

Diese Person soll nahestehend sein, sei ein Mitglied einer Familie oder ein Kollege bei der Arbeit oder einem Kurs, eine Person, mit der man mehrere Stunden am Tag verbringen kann, eine Person, mit der man sich nicht peinlich fühlt, wenn man einen Fehler macht. Um diese Methode erfolgreich $\mathrm{zu}$ sein, sollen Lernende in einer Mittelstufe oder in einem überdurchschnittlichen Niveau sein.

Aufgrund dieser Nähe wird es viele Gespräche zwischen Lernenden geben. Um nach einem geeigneten Wort in einem Gespräch zu suchen, wird dadurch der Wortbestand im Sprachgehirn geübt. Denn Sprachgehirn arbeitet und funktioniert besser mit Vernetzungen. Normalerweise trifft man am Anfang auf Probleme, man stottert, macht Fehler, aber dadurch profitiert man 
stark, vor allem vom Praktizieren des Sprechens. Auch wenn man einen Fehler macht, fühlt man sich nicht peinlich, da die Gesprächspartner nahestehend sind.

\section{Das Sprechen in zwei Sprachen zu Hause}

Um diesen Tipp durchzuführen, braucht man keine große Mühe. Wenn ein Lernender keinen Gesprächspartner in der Nähe findet, mit dem er immer auf Deutsch spricht, dann kann er mit einem in seiner Familie oder einem Kollegen bei der Arbeit oder einem Kurs vereinbaren, dass sie in zwei Sprachen Arabisch und Deutsch sprechen. Ein Lernender soll zuerst mit einem Gesprächspartner auf Deutsch und dann denselben Satz auf Arabisch wiederholen.

Dieser Lernstil bietet einem Lerneneden eine Möglichkeit jemanden finden, der ihm und seinem Gespräch in deutscher Sprache zuhört. Zwar verstehen die Gesprächspartner kein Wort von dem, was ein Lernender spricht, aber ein Gesprächspartner wird ihm zuhören, da ein arabischer Satz nach einem deutschen Satz kommt. Das wäre natürlich ein schöner Weg und ein schöner Trick, mit dem man die Sprechfertigkeit fördern kann.

\section{Selbstgespräche auf Deutsch}

Das ist die einfachste und leichteste Methode, da ein Lernender nicht verpflichtet ist, sein Gespräch mit jemandem zu teilen, sondern nur mit sich selbst sprechen kann. Das ist vielleicht seltsam. Aber wie kann man das tun? Es ist gewöhnlich zu sagen: Ich habe mit mir selbst gesprochen. Ja, eine Person spricht immer mit sich selbst, aber sie spricht oft still. Wörter und Sätze werden im Sprachgegirn verwendet, ohne dass sie Zunge und Lippen gelangen. Man hat das bestimmt so oft bei unterschiedlichen Sichtweisen zwischen ihm und sich selbst ausprobiert. Und da sich ein Gespräch im Innern dreht, dann gibt es eine Sprache, die in diesem Gespräch verwendet wird. Man muss nur die arabische Sprache gegen die deutsche Sprache austauschen.

Am Anfang ergeben sich Schwierigkeiten im Umgang mit diesem Tipp, aber man gewöhnt sich allmählich daran und am Ende profitiert viel davon. Es ist eine der wirksamen Methoden, die Sprachexperten empfehlen. Am Anfang soll man eine große Mühe gebenn, aber zu Ende wird man entdecken, dass man Deutsch spontan ohne Anstrengung sprcht.

Wenn man diese Methode anwendet, wird man feststellen, dass man bei einigen Wörtern ins Stocken gerät, deren Bedeutung auf Deutsch unklar ist. Dann sucht man nach der Bedeutung dieser Wörter, um diese dann zu lernen. So entwickelt man durch diesen Prozess seine Fremdsprache Deutsch und bereichert seinen Wortschatz.

Es ist eine schöne und nützliche Art, weil man spricht, ohne Angst vor Fehlern und Fallstricken zu haben. Das Einfache ist, wie wir immer sagen, das Geheimnis, jeden Tag in geringem Maße weiterzumachen

Wir sind der Meinung, dass der beste Weg, eine Sprache zu lernen, darin besteht, so bald wie möglich mit dem Sprechen zu beginnen. Aus diesem Grund bieten viele Websites und OnlineChats die Möglichkeit des Sprachaustauschs (in Form eines Sprachtandems). Der Wortschatzinhalt der MosaLingua Apps wurde daher speziell für den unmittelbaren Einsatz in Diskussionen ausgewählt. Die Mehrheit der Sprachportale schlägt ihren Benutzern vor, eine 
Webcam zu benutzen, um miteinander über bereits programmierte Sitzungen zu sprechen. Dies ist jedoch nicht die einzige Möglichkeit, ihre Sprachkenntnisse über Online-Chats zu verbessern.

\section{Babchat - Fremdsprachen online lernen}

Babchat ist ein kostenloser Online-Dienst, der den Chat des bekannten Sprachunternehmens Babbel 2014 fortsetzen soll. Dies geschieht in einem Sprach- oder Textchat, in den man einfach einsteigen kann. Man benötigt kein Babbel-Konto, da Babchat eine inoffizielle Ergänzung ist. Man kann einfach an Leute aus Deutschland, Österreich oder der Schweiz schreiben oder akzeptiert man eine Chat-Anfrage. Nun kann man in den Chat-Räumen auf verschiedene Sprachen schreiben und die eigenen Sprachkenntnisse verbessern.

\section{Das Sprechen mit der Außenwelt}

Das Internet, Computer, Smartphones und Technologien sind eine großartige Gelegenheit, die die Welt zu einem Dorf gemacht haben, den Osten mit dem Westen und den Norden mit dem Süden verbunden haben. Dadurch mischen sich Kulturen und konvergieren Sprachen. Jetzt kann man direkt und kostenlos mit Menschen aus weit entfernten Orten auf der Erde kommunizieren. Man kann also von diesen Technologien profitieren, die wir entwickelt haben, um unseren Traum, in anderen Sprachen zu sprechen, zu verwirklichen. Aus diesem Grund ist es einfach, dorthin zu gelangen und zu unterschiedlichen Zeiten sich mit Gefährten und Freunden in ihrer Muttersprache zu unterhalten.

Das Problem, auf das man jedoch leicht stoßen kann, ist, wie man diese ausländischen Freunde finden kann, und wer auch immer bereit ist und Lust darauf hat, von seiner Zeit für ein Gespräch mit Fremdsprachenlernenden zu opfern. Es mag ein einfaches Problem sein, aber es gibt viele Lösungen dafür. Es gibt viele Telefonanwendungen und Websites, die die Möglichkeit bieten, Sprachlerner miteinander zu verbinden. Man möchte z.B. Deutsch oder Englisch lernen und es gibt eine andere Person auf der anderen Seite der Welt, die die arabische Sprache lernen möchte. Durch diese Programme findet man solche Freunde und eine gegenseitige Hilfebeziehung bauen. Beide versuchen bei der Entwicklung der gewünschten Sprache zu helfen.

\section{Das Verbreiten der Stimme online}

Dies ist ein professioneller und besonders nützlicher Schritt für einen Fremdsprachenlernenden. Wenn man einen großen Schritt nach vorne machen möchte, dann könnte man mit Video- oder Audio-Publishing beginnen. So kann man auf Deutsch entweder die Sound Cloud-Website und die Anwendung für Audio-Publishing oder die (YouTube- Video-Publishing-Site) verwenden und Unterrichtsstunden in jedem Bereich veröffentlichen. Es ist eines der Felder, die man beherrschen kann, z. B. das Erklären von Computerprogrammen unter anderem, dies jedoch auf Deutsch. Da das Feld ein Fachgebiet und eine Spezialisierung ist, ist es einfach, weil man viele Begriffe in diesem Fachgebiet lernen kann. Wenn diese Methode unerfüllbar ist, kann man seine Stimme über die SoundCloud-Plattform aufnehmen, um sie in Zukunft selbst zu hören. So kann man versuchen, seine Stimme aufnehmen und regelmäßig auf dieser Website über verschiedene Dinge zu sprechen. Man kann lautstark über Mike mit sich selbst sprechen und seine Stimme aufnehmen, damit man noch nach Monaten sich selbst zuhören kann, um sich an seine Übungen 
zu erinnern und zu sehen, wie das eigene Niveau damals war und wo man jetzt angekommen ist. Natürlich kann man die Datenschutzeinstellungen beim Veröffentlichen so ändern, dass nur man selbst seine Datensätze anhören kann.

\section{Das Abonnieren von einigen kostenpflichtigen Diensten}

Das ist ein kostenpflichtiger Dienst. Wenn man genug Geld hat und bereit ist, einen Teil davon auszugeben, um sein Deutschniveau zu verbessern, helfen diese kostenpflichtigen Dienste erheblich. Dadurch kann man Konversationsstunden mit ausländischen Sprechern und professionellen Lehrern kaufen und so viel mit ihnen sprechen, wie man möchtet. So wird die finanzielle Entschädigung pro Stunde berechnet, und der Stundensatz variiert je nach Standort und je nach Lehrer selbst. Es gibt viele dieser Websites und Dienste

\section{Schlussfolgerung}

Die Fremdsprachenkenntnisse gewinnen mit dem globalen Einfluss und der zunehmenden Mobilität der Menschen an Bedeutung, und Mehrsprachigkeit wird zum Ziel moderner Bildung genommen. Der Umgang mit Fremdsprachen und Kulturen wird heutzutage als eine wesentliche Qualifikationsanforderung am Arbeitsmarkt angesehen.

Infolgedessen hat der Fremdsprachenunterricht in letzter Zeit einige Änderungen vollzogen. Das Ziel des Unterrichts sind weder die Fortschritte in der grammatikalischen Struktur einer Fremdsprache noch "Musterübungen", sondern sogenannte Kommunikationsfertigkeit, d. h. die Fähigkeit, mit anderen Menschen zu kommunizieren. Da Austausch von Infomation durch Sprechen (oder Schreiben) realisiert wird, hat sich mit dieser Anforderung auch die Bedeutung des Sprechens im Fremdsprachenunterricht geändert, und deren Entwicklung im Klassenzimmer sollte mehr Beachtung geschenkt werden.

In der Tat ist dies jedoch nicht immer der Fall. Aus meinem Beitrag geht hervor, dass ich versucht habe, die Möglichkeiten aufzuzeigen, die Sprechfertigkeit im DaF-Unterricht zu verstärken, und vor allem einige konkrete kommunikative Fähigkeiten und Fertigkeiten zu empfehlen, die im DaF-Unterricht mit Studierenden in Sprachenfakultät verwendet werden können.

Aus dem theoretischen Teil haben wir gefolgert, dass sich die Bedeutung der Sprechfertigkeit und sich die Methode im Laufe der Zeit verändert hat und die mündliche Kommuniation im Fremdsprachenunterricht von größter Bedeutung ist.

So kann dieser Beitrag sowohl als Material meiner eigenen Erfahrung als auch als Anregung für andere LehrerInnen für weitere Sprechaktivitäten dienen.

Unterdessen sollte er auch darauf hinweisen, dass die Förderung der Sprechfertigkeit in der Sprachenfakultät in Bagdad von Erfolg gekrönt sein kann. Auch bei gelegentlichem Einbeziehen von Sprechaktivitäten in den Unterricht sollte, wie die Ergebnisse der Fragebögen zeigten, die Sprechfähigkeit so komplex gefördert werden, dass bei den Studierenden ein möglichst hoher Automatisierungsgrad erreicht wird und die Studierende jederzeit in der Lage sind, sich auszudrücken, um die konkrete Kommunikationssituation zum geübten Thema zu verstehen. 


\section{Bibliografie}

- Alhachami, Qasim (2016): Das gesungene Wort im Deutsch-als-FremdspracheUnterricht Eine empirische Studie an der Deutschabteilung der Sprachenfakultät, Mannheim.

- Birkenbihl, Vera F. (2013) Stroh im Kopf?: Vom Gehirn-Besitzer zum GehirnBenutzer, München

- Grohnfeldt, Manfred 332: 2007): Störungen der Sprachverarbeitung332 in Manfred Grohnfeldt Lexikon der Sprachtherapie (Hrsg) 2007. 332-334

- Krumm H-J, Fandrych C, Hufeisen B, Riemer C (Eds) (2010): Deutsch als Fremdund Zweitsprache. Ein Internationales Handbuch Handbücher zur Sprach- und Kommunikationswissenschaft/HSK, 2. und vollständig überarbeitete und neu zusammengestellte Auflage.Berlin/New York: de Gruyter.

- Staiger, Michael (2014) Audiovisuelle Medien im Deutschunterricht.in: Volker Frederking/Axel Krommer/Thomas Möbius (Hg.): Deutschunterricht in Theorie und Praxis. Bd 8. 236-268).

\section{Anhang}

\section{Fragebögen}




\section{Fragebogen}

Liebe Studierende,

mein Name ist Dr. Qasim Jebur Abrah. Ich arbeite als Deutschlehrer an der

Übersetzungsabteilung/Philosophische Fakultät/ Universität Wasit. Im Rahmen meines

Beitrages „Die Sprechfertigkeit im DaF-Unterricht",, benötige ich Ihre Meinung als

Zielvorgabe. Ich kann Ihnen versichern, dass Ihre Daten nur für wissenschaftliche und

akademische Zwecke verwendet werden.

Fakultät:

Studienjahr: .................................... Geschlecht:

Bermerkung: Übertreiben Sie bitte nicht beim Ausfüllen des Formulars und beschreiben Sie bitte, was Sie sich fühlen und richtig leisten können!

\section{مطلوب**}

1.

$$
\text { * عنوان بريد إلكثروني }
$$

2. Vollname: *

3. 1. Wie können Sie Ihr Sprachniveau einschätzen? *

حدد دائرة واحدة فقط.

Sehr gut

Gut

Ausreichend

Schlecht

Unzureichend

Schätzen Sie Ihre Sprechfertigkeit anhand der folgenden Punkte ein (ankreuzen, wenn "ja", und wenn "nein")! 
8. 7. Ich kann Träume, Hoffnungen und Ziele beschreiben, eine Geschichte erzählen und mich ohne Vorbereitung an Gesprächen über vertraute und interessante Themen teilnehmen. *

حدد دائرة واحدة فقط.

$\mathrm{Ja}$

Nein

9. 8. Ich kann ein einfaches direktes Gespräch beginnen, in Gang halten und beenden und kann meine Reaktion auf einen Text oder ein Buch, einen Film oder eine Aussage, die ich gelesen habe, beschreiben. *

حدد دائرة واحدة فقط.

$\mathrm{Ja}$

Nein

10. 9. Ich kann mich aktiv an längeren Gesprächen über die meisten Themen teilnehmen und dabei einige komplexe Satzstrukturen verwenden, ohne blitzschnell nach Worten zu suchen. *

حدد دائرة واحدة فقط.

Ja

Nein

11. 10. Nutzen Sie soziale Medien in Ihrem Alltag, um Ihr Deutsch zu verbessern und zu entwickeln? *

حدد دائرة واحدة فقط.

Ja

Nein 
12. 11. Haben Sie schwierigkeiten beim Umgang mit sozialen Medien? *

حدد دائرة واحدة فقط.

Ja

Nein

13. 12. Hören Sie Deutschlandfunk Nachrichten? *

حدد دائرة واحدة فقط.

Ja

Nein

14. 13. Wie oft folgen Sie Tagesnachrichten und Hintergrundberichten auf Deutsch?

حدد دائرة واحدة فقط.

Selten

sehr oft

Oft

Niemals

15. 14. Folgen Sie Deutsche Filme und Serien? *

حدد دائرة واحدة ققط.

Ja

Nein 
16. 15.Unterhalten Sie mit Ihrem Partner/Ihrer Partnerin im Klassenraum, in der Pause oder außerhalb der Universität auf Deutsch? *

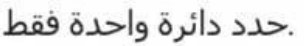
$\mathrm{Ja}$
Nein

17. 16. Unterhalten Sie mit deutschsprachigen Personen im In- und Ausland? *

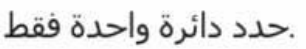
$\mathrm{Ja}$
Nein

18. 17. Sprechen Sie mit sich selbst auf Deutsch? *

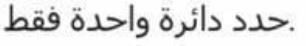

$\mathrm{Ja}$

Nein

\footnotetext{
Google.
}

\section{Google نماذجن}

